\title{
Metodologia de Estudo de Dieta Total no Estado de São Paulo para estimativa de ingestão dietética de elementos tóxicos e essenciais: a elaboração da Cesta de Mercado (Market Basket)
}

\author{
Total diet study in the state of São Paulo, Brazil, to estimate the dietary intakes \\ of toxic and essential elements: development of the Market Basket
}

Roseane Pagliaro AVEGLIANO ${ }^{1,2 *}$, Vera Akiko MAIHARA², Fábio Fernando da SILVA ${ }^{3}$

\begin{abstract}
Resumo
O objetivo do estudo foi elaborar a Cesta de Mercado para a realização de Estudos de Dieta Total (EDT) para avaliação de ingestão dietética de elementos tóxicos e essenciais no Estado de São Paulo. A fonte de dados foi a pesquisa de consumo nacional domiciliar atualizada, Pesquisa de Orçamentos Familiares (POF) 2002-2003, do Instituto Brasileiro de Geografia e Estatística (IBGE). Para estimar o consumo de alimentos pela população estudada, os dados de aquisição alimentar utilizados foram pertinentes ao Estado de São Paulo. A Cesta de Mercado inicialmente foi formulada com os dados disponíveis das publicações do IBGE, e para detalhamento dos alimentos foram pesquisados os seus Micro dados, resultando numa Cesta de Mercado com 71 alimentos, que corresponde a $72 \%$ do peso dos alimentos da população do Estado de São Paulo, segundo a POF. Como abordagem sugerida pelos EDT já realizados em outros países, os alimentos foram agrupados em 30 compostos, que serão posteriormente analisados para determinar o seu conteúdo dos elementos essenciais e tóxicos.

Palavras-chave: Cesta de Mercado; estudos de dieta total; pesquisa de orçamentos familiares.
\end{abstract}

\begin{abstract}
The objective of this paper was to establish the Market Basket for the Total Diet Study (TDS) in the state of São Paulo - Brasil in order to assess toxic and essential element dietary intakes. The food consumption data source used was from a recent household food budget survey, the "Pesquisa de Orçamento Familiar (POF) 2002-2003", conducted by the Brazilian Institute for Geography and Statistics (Instituto Brasileiro de Geografia e Estatística). The Market Basket formulation was based on information from publications of the Brazilian Institute for Geography and Statistics. Food details were researched in the POF Microdata, which resulted in a Market Basket containing 71 items. This corresponds to $72 \%$ of the food intake weight for the São Paulo State population. Foods were grouped in 30 compounds, which will be analyzed to determine essential and toxic element contents, according to suggested TDS approaches already carried out in other countries.

Keywords: Market Basket; total diet study; household food budget survey.
\end{abstract}

\section{Introdução}

O Estudo de Dieta Total (EDT) ou Cesta de Mercado (Market Basket) é recomendado pela World Health Organization (WHO) como o método mais adequado para estimar a ingestão dietética de elementos químicos e de nutrientes através de análises diretas em amostras de alimentos preparados, como os prontos para o consumo (table-ready), previamente definidos em uma Cesta de Mercado. Essa Cesta deve ser constituída por alimentos ou grupos de alimentos que reflitam os hábitos dietéticos médios de grandes grupos populacionais. Os alimentos amostrados devem ser preparados, pois o preparo pode alterar a concentração de certos elementos químicos, como por exemplo, alguns contaminantes presentes nos alimentos crus que podem ser dissolvidos, volatizados ou degradados, e a concentração de alguns metais pode aumentar pelo contato com utensílios de cozinha (WHO, 1985).
A comparação dos EDT com os valores regulatórios nacionais e internacionais torna-se essencial para avaliar se uma dieta nacional é segura (WHO, 1999; 2002c; 2004).

A WHO incentiva todos os países a conduzirem seus próprios EDT, uma vez que em cada país a dieta e os hábitos alimentares são diferentes, e com essa finalidade tem patrocinado workshops internacionais e regionais sobre EDT, desde 1999. A cada workshop há um crescente número de países que apresentam seus próprios EDT (WHO, 2002a,b,c; 2004;).

Os dados de consumo nacionais devem ser usados sempre que possível, pois os EDT geralmente são elaborados para determinar a ingestão de contaminantes em uma dieta nacionalmente representativa, incorporando todas as regiões geográficas de um país (WHO, 1999; 2002b). Porém, do ponto de vista prático, é

${ }^{1}$ Divisão de Alimentação, Coordenadoria de Assistência Social - Coseas, Universidade de São Paulo - USP, Rua do Anfiteatro, 295, CEP 05508-900, São Paulo, SP - Brasil, E-mail: pagliaro@usp.br

2 Instituto de Pesquisas Energéticas e Nucleares - IPEN, Comissão Nacional de Energia Nuclear - CNEN-SP, Universidade de São Paulo - USP, Av. Prof. Lineu Prestes, 2242, CEP 05508-000, São Paulo, SP - Brasil

${ }^{3}$ Empresa Júnior de Informática, Matemática e Estatística - IME Jr, Universidade de São Paulo - USP, Rua do Matão, 1010, Sala 258-A, CEP 05508-090, São Paulo, SP - Brasil

${ }^{*}$ A quem a correspondência deve ser enviada 
mais realista uma amostragem seletiva de alguns centros do país; se necessária, direcionada para segmentos selecionados da população (WHO, 1985) em que os códigos de alimentos de pesquisas nacionais sejam mapeados em grupos de alimentos de interesse para essa população restrita.

Os dados de consumo fornecidos por pesquisas de consumo nacionais são amplos, refletem os padrões de consumo alimentares típicos e abordam médias de consumo da ingestão de alimentos. Esses dados devem ser tão recentes quanto possível e definidos a cada estudo, em virtude da possibilidade de mudanças rápidas dos padrões dietéticos locais (WHO, 2002b; 2004).

Uma vez que os EDT estimam a ingestão dietética total dos elementos pesquisados e não apenas relatam a quantidade deles em um único alimento (STENHOUSE, 1993), são particularmente valiosos em determinar se as substâncias químicas são amplamente distribuídas entre todos os tipos dos principais alimentos ou restritas a alguns tipos de alimentos, como frutas, vegetais, dentre outros (WHO, 1985).

Turrini e Lombardi-Boccia (2002) destacam a importância da etapa da coleta de dados dos alimentos, pois quanto mais detalhada a lista de alimentos, mais representativa é a Cesta de Mercado.

Portanto, os EDT são complexos, para a sua elaboração necessita-se de técnicas avançadas de instrumentação em relação à infra-estrutura de laboratórios, tornando-se caros, sobretudo para os países em desenvolvimento (WHO, 1999; 2002b).

Há duas diferentes abordagens para os alimentos, na realização de um EDT:

- análise individual de alimentos, na qual pode haver um alimento representativo para cada grupo de alimentos, como por exemplo, tangerina representando todas as frutas cítricas e seus resultados analíticos em relação à concentração de substâncias químicas tóxicas usadas para consumo do grupo de alimentos (WHO, 1999). Essa abordagem requer um grande número de amostras; e

- análise de compostos de grupo de alimentos, combinandose alimentos similares na mesma proporção (\%) relativa de cada um dos alimentos, que os obtidos através das pesquisas de consumo(MUNÕZ et al., 2005; WHO, 1985; 1999).

A abordagem por compostos tem como vantagem o número reduzido de amostras para análise (WHO, 1985; 1999). Portanto, uma maneira de gerar uma estimativa confiável de exposição média aos contaminantes alimentares com menor custo. Por outro lado, a concentração de contaminantes é diluída entre os componentes e pode resultar em níveis abaixo dos detectáveis (efeito-diluição), podendo mascarar uma fonte de contaminação (WHO,1985).

Os resultados analíticos das amostras de alimentos, tanto na análise individual como em compostos, são combinados com informações de consumo do grupo populacional para estimar ingestões dietéticas dos elementos químicos analisados.

\section{Material e métodos}

Os dados de aquisição alimentar para a formulação da Cesta de Mercado foram extraídos da Pesquisa de Orçamentos Familiares (POF) 2002-2003, a mais recente do Instituto Brasileiro de Geografia e Estatística (IBGE), realizada entre julho de 2002 e junho de 2003.

A POF 2002-2003 propicia resultados para as grandes regiões do Brasil e para as Unidades da Federação, incluindo as áreas urbana e rural. Para o Estado de São Paulo, gerou-se uma amostra de 2017 domicílios, cujos moradores compartilhavam da mesma fonte de alimentação ou as despesas com moradia.

A POF sendo uma pesquisa de múltiplos usos, embora não corresponda exatamente aos modelos de levantamentos para o estudo de avaliação alimentar, fornece a observação de um amplo conjunto de variáveis na área da alimentação, dentre elas as de aquisição de alimentos. As quantidades adquiridas de alimentos e bebidas revelam, com alto grau de detalhamento, os hábitos alimentares dos brasileiros, segundo os regionalismos. Nesse sentido, dados sobre aquisição de alimentos levantados numa POF podem fornecer informações úteis sobre o padrão alimentar das famílias, desde que a fração não aproveitada dos alimentos não varie muito de alimento para alimento e que aqueles consumidos fora do domicílio representem uma proporção reduzida do consumo alimentar total das famílias.

A informações referentes aos alimentos e bebidas adquiridos pelas famílias dos domicílios selecionados foram pesquisadas segundo o período de referência de 7 dias consecutivos, com informações do registro diário, resultando para o Estado de São Paulo, em descrições agrupadas em 326 alimentos.

A elaboração da Cesta de Mercado (Market Basket) foi dividida nas etapas a seguir:

\subsection{Definição inicial da Cesta de Mercado}

Foram analisados os dados referentes ao Estado de São Paulo e disponibilizados na Tabela 1.4 - Aquisição alimentar domiciliar per capita anual, por Unidades da Federação, segundo os produtos - Região Sudeste - período 2002-2003, da publicação "Aquisição Alimentar Domiciliar per capita" da POF IBGE 2002-2003 (IBGE, 2004a).

Desta Tabela foram extraídos os dados para a Cesta de Mercado, sendo que para se obter o per capita diário dividiu-se o valor per capita anual por 364 dias, correspondente ao número de dias de um ano, conforme informação da POF, e transformando a unidade inicial quilos $(\mathrm{kg})$ na unidade gramas $(\mathrm{g})$.

Partindo-se deste resultado, definiram-se os alimentos a serem considerados para a Cesta de Mercado, baseando-se no critério da percentagem de representatividade dos alimentos adquiridos para consumo diário. Esta representatividade foi obtida pela percentagem do peso em gramas dos per capitas diários dos alimentos selecionados para a Cesta de Mercado em relação ao peso total dos per capitas diários dos 326 alimentos mencionados na Tabela 1.4 (IBGE, 2004a).

A definição para a escolha dos alimentos da Cesta de Mercado do presente estudo foi baseada nas percentagens utilizadas 
em EDT realizados em outros países, que variavam entre 70\% (EDT da Nova Zelândia (VANNORT; CRESSEY; SILVERS, 2000 ) a mais de $95 \%$ do peso da dieta (EDT da República Tcheca (WHO, 2002b) e França (WHO, 2004) e no valor per capita diário em gramas da POF para o Estado de São Paulo.

Foram então consideradas quatro alternativas:

Alternativa 1: Usando o critério de alimentos adquiridos em quantidades superiores a 1 grama per capita diário, segundo a Tabela 1.4, 123 alimentos seriam incluídos, representando 93,8\% do peso dos 326 alimentos da POF. Este critério de escolha de consumo superior a 1 grama/dia, representando mais de $90 \%$ da dieta, baseou-se em EDT realizados na França (WHO, 2004).

Esta alternativa seria indicada para este trabalho em razão de sua alta representatividade, porém, devido ao elevado número de amostras para análise direta no laboratório poderia se tornar inviável.

Alternativa 2: Usando o critério de alimentos adquiridos em quantidades superiores a $7 \mathrm{~g}$ per capita diário, segundo a Tabela 1.4, 26 alimentos seriam incluídos, representando 66,3\% do peso dos 326 alimentos da POF. Esta percentagem foi considerada baixa, pois o valor mínimo encontrado na literatura consultada era de $70 \%$.

Alternativa 3: Usando o critério de alimentos adquiridos em quantidades superiores a $5 \mathrm{~g}$ per capita diário, segundo a Tabela 1.4, 35 alimentos seriam incluídos, representando $72 \%$ do peso dos 326 alimentos da POF. Esta alternativa poderia ter sido escolhida por estar acima do valor mínimo na literatura consultada, porém, ela excluiria alguns alimentos do hábito alimentar no Estado de São Paulo. Então, optou-se por escolher um maior número de alimentos para tornar a Cesta de Mercado mais representativa.

Alternativa 4: Usando o critério de alimentos adquiridos em quantidades superiores a $2 \mathrm{~g}$ per capita diário, segundo a Tabela 1.4, 79 alimentos seriam incluídos, representando 87,3\% do peso dos 326 alimentos da POF.

A alternativa 4 foi escolhida por representar percentagem de alimentos acima da percentagem mínima nos EDT na literatura consultada, por contemplar alimentos do hábito alimentar no Estado de São Paulo e porque o número de alimentos a ser analisado era viável.

Os 326 alimentos da POF estão agrupados em 16 grupos e 45 subgrupos, sendo que neste trabalho utilizou-se o critério da classificação dos alimentos da POF em subgrupos, por refletirem melhor as diferentes categorias dos alimentos, isto é, a heterogeneidade interna.

Para finalizar a definição da Cesta de Mercado composta por 79 alimentos, a mesma foi acrescida de 4 alimentos pertencentes aos subgrupos dos peixes de água salgada (4 tipos) e água doce (1 tipo), para a mensuração de elementos tóxicos. Esse acréscimo baseou-se na literatura pesquisada, e podem ser incluídos nos EDT alimentos que conhecidamente podem conter altos teores dos elementos tóxicos. Embora a Tabela 1.4 registrasse aquisição menor que $2 \mathrm{~g}$ diários para esses 5 alimentos, eles foram incluídos pela importância de seus teores de arsênio e mercúrio. Esses
5 tipos de peixes correspondem aos de maior valor de aquisição, nos respectivos subgrupos. Com a inclusão do per capita dos peixes, o peso da Cesta de Mercado passaria a representar 87,5\% do peso dos 326 alimentos da POF.

Os 84 alimentos selecionados para a Cesta de Mercado pertencem a 32 subgrupos. O número de alimentos de cada subgrupo da Cesta de Mercado e a proporção deles em relação ao total de alimentos do mesmo subgrupo constante na POF varia. Em 11 subgrupos não foi encontrado nenhum alimento com aquisição superior a $2 \mathrm{~g}$ diários e 2 subgrupos dos peixes foram contemplados pelas razões já citadas.

Após a escolha inicial dos 84 alimentos e dos critérios definidos, baseando-se na Tabela 1.4, que disponibiliza informações em 326 alimentos agrupados, recorreu-se às informações adicionais disponibilizadas pelo IBGE através de um banco de dados denominado Micro dados da POF 2002-2003 do IBGE (2004b), contendo para o Estado de São Paulo 5804 alimentos individualizados.

Sobre esses 84 alimentos, não havia informações suficientes para a sua correta identificação. Para melhor defini-los foi necessário recorrer às informações dos Micro dados.

A utilização desse recurso adicional teve a finalidade de aprimorar as especificações e quantidades dos alimentos, para que as coletas dos mesmos para as análises diretas fossem as mais precisas possíveis.

Os Micro dados permitem a subdivisão dos tipos dos alimentos, sendo que dessa forma a soma dos per capita diários dos 326 alimentos e dos 5804 é a mesma, ou seja, $938 \mathrm{~g}$.

\subsection{Detalhamento da Cesta de Mercado}

Com base nos Micro dados foram selecionadas as seguintes variáveis, segundo o registro da Caderneta de Despesa Coletiva da POF:

- Tipo de registro;

- Código da UF;

- Fator expansão 2 (domićlio);

- Número do grupo (Micro dados);

- Código do item;

- Fator de anualização; e

- Quantidade em kg.

Segundo o objetivo deste trabalho, selecionaram-se dados referentes a alimentos e ao Estado de São Paulo. Para isto, selecionou-se o Tipo de Registro e o Código da UF.

Para a identificação dos alimentos - Itens e Séries - nos Micro dados, se faz necessária a composição de um código (código concatenado), que será formado pela união da variável Número do grupo (Micro dados) com a variável Código do item. O Código do item discrimina especificamente cada alimento. Contudo, também se faz referência a uma série de alimentos. A identificação das séries, segundo o IBGE, é feita extraindo-se os 5 primeiros dígitos do código concatenado dos alimentos. 
Portanto, um alimento é identificado pelo seu próprio código e também possui um código referente à série em que ele foi alocado.

Para a obtenção do valor referente à Aquisição Alimentar per capita de cada alimento, aplicou-se o Fator de Anualização e o Fator de Expansão 2 (domicílio) sobre a quantidade em kg do alimento. Sendo assim, para cada alimento (código concatenado) aplicou-se (multiplicação) o fator de anualização e o fator de expansão 2. Em seguida, somaram-se todos os valores obtidos para cada alimento, formando assim, a aquisição alimentar total anual do alimento, em kg. Para o valor da aquisição alimentar anual per capita $(\mathrm{kg})$, dividiu-se o valor obtido para cada alimento pela estimativa populacional em questão, que no estudo se refere ao Estado de São Paulo e equivale a 38.465.675 habitantes, segundo o IBGE (2004a). Para o valor diário da aquisição alimentar per capita em gramas (g), dividiu-se o valor da aquisição alimentar anual per capita $(\mathrm{kg})$ por 364 (total de dias em um ano, referente aos critérios do IBGE) e este resultado foi multiplicado por 1.000, convertendo-se assim, a unidade de quilos $(\mathrm{kg})$ para gramas $(\mathrm{g})$.

Os valores referentes às séries de alimentos foram obtidos somando-se os valores de aquisição alimentar per capita de cada alimento que compõe a específica série.

Dos 5804 alimentos dos Micro dados, selecionaram-se os de aquisição superior a $2 \mathrm{~g}$, resultando 75 alimentos. Ainda nesta etapa foram eliminados 8 dos 75 alimentos descritos como "não especificados", independentemente do per capita diário em gramas, resultando em 67 alimentos. Para finalizar a Cesta de Mercado, a mesma foi acrescida dos peixes, em subgrupos de água salgada (3 tipos) e de água doce (1 tipo). Escolheramse os peixes mais consumidos, cujos registros identificavam devidamente o alimento.

\subsection{Amostragem}

A abordagem utilizada será a de agrupar os alimentos em compostos de alimentos ao invés de alimentos individuais.

Os alimentos pertencentes a cada subgrupo formam o composto. O número de compostos é o número de subgrupos, que corresponde a 30. Assim haverá 30 amostras no total.

Os alimentos serão posteriormente preparados, uma vez que os dados referentes aos alimentos da Cesta de Mercado indicam o peso dos mesmos tal como foram adquiridos, e o EDT utiliza alimentos prontos para consumo.

Definiu-se que cada composto, com os alimentos preparados, deverá pesar $1 \mathrm{~kg}$, quantidade suficiente para que possam ser realizadas as análises posteriores.

Depois do preparo, os alimentos serão pesados e estarão presentes nos compostos de $1 \mathrm{~kg}$, nas mesmas proporções em que aparecem nos subgrupos da Cesta de Mercado.

\section{Definição da quantidade de alimentos a ser coletada}

Os alimentos serão coletados seguindo-se o tipo de alimento definido na Cesta de Mercado, na variedade disponível no local da coleta.
Para assegurar que os alimentos sejam coletados em quantidade suficiente para que, após o preparo, resultem nas quantidades determinadas pela sua percentagem no composto de $1 \mathrm{~kg}$, é necessário que se façam ajustes nas quantidades definidas na Cesta de Mercado. As variações de peso ocorrem devido à exclusão de partes não comestíveis e ao ganho ou à perda de peso dos alimentos durante o preparo. São ajustes necessários:

- transformar o peso do alimento pronto para o consumo, definido no composto, em peso líquido do alimento cru, aplicando-se o Indicador de Conversão (IC). O IC representa a mudança do estado do alimento, caso o alimento conste no EDT como alimento cozido, sendo IC = peso do alimento cozido/peso do alimento cru. Outros ajustes ocorridos nas transformações dos alimentos, além de cocção, precisam ser realizados, por exemplo, a infusão será transformada em café em pó; e

- transformar o peso líquido do alimento cru em peso bruto, pelo Indicador de Parte Comestível (IPC), sendo IPC = peso bruto $(\mathrm{g}) /$ peso líquido $(\mathrm{g})$.

Os índices IPC e IC foram obtidos em Phillippi (1994; 1995; 1999), Phillippi et al. (1999) e Ornellas (2001), sendo que alguns índices inexistentes na literatura foram definidos na prática utilizando-se alimentos do restaurante onde se realizariam os preparos dos alimentos da Cesta de Mercado.

A aplicação desses índices serve como uma orientação para obtenção das quantidades estimadas dos alimentos a serem coletadas.

\section{Preparo dos alimentos da Cesta de Mercado}

O preparo será feito no mesmo local da coleta, podendo variar entre o mais simples, como lavagem e descascamento, até a cocção, seguindo um guia de preparação previamente estabelecido para este estudo.

De uma maneira geral, os alimentos que são consumidos cozidos serão preparados sem a utilização de temperos e condimentos, e utilizando-se métodos de cocção que não introduzam outros ingredientes exceto a água, a fim de proporcionar resultado mais preciso nas análises diretas. Os métodos de cocção como: grelhar, assar em forno e cozinhar a vapor serão utilizados para evitar a introdução de óleos e gorduras na preparação.

Será utilizada água de torneira ao invés de água destilada para garantir que os elementos tóxicos e essenciais que possam estar presentes na água sejam considerados na estimativa total de exposição dietética.

A água para a cocção dos alimentos e para o preparo do café deverá ser considerada como um integrante do EDT.

O alimento que pode ser consumido cru ou cozido (como por exemplo, o tomate) será incluído cru, para maximizar qualquer presença de elementos químicos.

Serão anotados os pesos dos alimentos durante as transformações do peso bruto em peso líquido e do peso cru em peso cozido, para a definição dos índices próprios dos alimentos (IPC e IC) da Cesta de Mercado. 
A utilização destes indicadores dos alimentos da Cesta de Mercado dará ao EDT a precisão nas informações das quantidades, evitando-se as estimativas ou aproximações com o uso de indicadores existentes na bibliografia consultada sobre técnicas dietéticas. Além disso, poderão ser utilizados na interpretação dos resultados após as análises laboratoriais ou em análises adicionais, caso seja necessária a repetição por algum erro na amostragem, como por exemplo, quantidade insuficiente de alimento coletado para as amostras.

\section{Resultados e discussão}

A metodologia de definição da Cesta de Mercado é um dado até então inexistente no Brasil, sendo que é o método mais adequado para estimar a ingestão dietética de contaminantes e nutrientes para grandes grupos populacionais. A Tabela 1 apresenta a composição da Cesta de Mercado elaborada segundo a metodologia do EDT a partir da POF 2002-2003 do IBGE, em quantidade per capita do alimento adquirido na população amostral do Estado de São Paulo, o agrupamento dos alimentos em compostos, a composição de cada composto e a quantidade do alimento a ser coletado para preparo para o EDT. Desta maneira, definiu-se então a Cesta de Mercado, que contém 71 alimentos, conforme descritos na Tabela 1. Os alimentos que a compõem são os mais representativos no Estado de São Paulo. Eles correspondem a $72 \%$ do peso dos 5804 alimentos da POF neste Estado.

A metodologia apresentada neste estudo baseou-se em EDT conduzidos em outros países, que basicamente consideraram as pesquisas nacionais de consumo como fonte de dados. As abordagens foram feitas por compostos de grupos de alimentos em países, como os EUA (até 1980) (EGAN, 2002; PENNINGTON, 1983; WHO, 1999; 2002b; 2004; U.S. FOOD AND DRUG ADMINISTRATION, 2003), Nova Zelândia (até 1982) (BRINSDON, 2002; NEW ZEALAND FOOD SAFETY AUTHORITY, 1997; 2005; 2006; VANNORT; CRESSEY; SILVERS, 2000; WHO, 1999; 2002b; 2004), Canadá (WHO, 1999; 2002b; 2004), China (WHO, 1999; 2002b; 2004), Chile (MUNÕZ et al., 2005), Espanha (WHO, 1999; 2004) e França (WHO, 2002b; 2004). A abordagem por alimentos individuais ou representativos foi utilizada nos EDT dos EUA (desde 1982) (EGAN, 2002; PENNINGTON, 1983; WHO, 1999; 2002b; 2004; U.S FOOD AND DRUG ADMINISTRATION, 2003), Nova Zelândia (desde 1987) (BRINSDON, 2002; NEW ZEALAND FOOD SAFETY AUTHORITY, 1997; 2005; 2006; VANNORT; CRESSEY; SILVERS, 2000; WHO,1999; 2002b; 2004), Austrália (FOOD STANDARDS AUSTRALIA NEW ZEALAND, 2003; 2005; WHO, 2002b), República Tcheca (WHO, 1999; 2002b; 2004) e Itália (LOMBARDI-BOCCIA et al. 2003; TURRINI; LOMBARDI-BOCCIA, 2002). Os EDT apresentaram variações nos números de alimentos ou compostos, citando-se entre eles: EUA, 280 alimentos (EGAN, 2002; PENNINGTON, 1983; WHO, 1999; 2002b; 2004; U.S. FOOD AND DRUG ADMINISTRATION, 2003); Austrália, 65 alimentos (FOOD STANDARDS AUSTRALIA NEW ZEALAND, 2003; 2005; WHO, 2002b); Nova Zelândia, 121 alimentos (BRINSDON, 2002; NEW ZEALAND FOOD SAFETY AUTHORITY, 1997; 2005; 2006; VANNORT; CRESSEY; SILVERS, 2000; WHO,1999; 2002b; 2004); Itália, 191 alimentos (LOMBARDI-BOCCIA et al., 2003; TURRINI; LOMBARDI-BOCCIA, 2002); Chile, 17 compostos (MUNÕZ et al., 2005) e Canadá, 140 compostos (WHO, 1999; 2002b; 2004).

\section{Conclusões}

A contribuição original deste trabalho evidencia-se na relevância da realização de EDT no Brasil para estimativas de ingestão dietética dos elementos tóxicos e essenciais, pois embora o país se inclua como participante nas atividades do Sistema de Monitoração Ambiental Global - Monitoramento de Contaminação de Alimentos e Programa de Avaliação (GEMS/ Food) (WHO, 1997)e em workshops de EDT, não tem apresentado dados, uma vez que são inexistentes.

A Cesta de Mercado do Estado de São Paulo é um instrumento valioso para conhecer a concentração de elementos tóxicos e essenciais pela população estudada, a fim de garantir uma alimentação segura, determinar a avaliação de riscos e comparar com os valores de referência nacionais e internacionais.

\section{Agradecimentos}

Ao CNPq (Projeto no 401994/2005-8) e à FAPESP (Projeto $n^{\circ}$ 2003/10156-6), pelo apoio financeiro e à professora doutora Rosa Maria Godoy Serpa da Fonseca - coordenadora da Coseas/ USP, pela colaboração.

\section{Referências bibliográficas}

BRINSDON, S. Food list 2003/04 New Zealand Total Diet Survey. Wellington: New Zealand Food Safety Authority, 2002. Disponível em: <http://www.nzfsa.govt.nz/science/research-projects/totaldiet-survey/food-list-2003/food-list-2003.pdf.>. Acesso em: 9 jul. 2006.

EGAN, K. FDA's Total Diet Study: monitoring U.S. food supply safety. Rockville, M.D.: U.S Food and Drug Administration, 2002. Disponível em: <http://vm.cfsan.fda.gov/ dms/tdsoview.html>. Acesso em: 20 out. 2004.

FOOD STANDARDS AUSTRALIA NEW ZEALAND. The 20th Australian Total Diet Survey: a total diet survey of pesticide residues and contaminants. 2003.Disponível em: <http://www. foodstandars.gov.au/srcfiles/final_20th_Total_Diet_Survey.pdf.> Acesso em: 22 jan. 2006.

The 21st australian Total Diet Study: a total diet study of sulphites, benzoates and sorbates. 2005. Disponível em: <http://www.foodstandars.gov.au/mediareleasespublications/ publications/21staustralia.> Acesso em: 22 jan. 2006.

IBGE. Instituto Brasileiro de Geografia e Estatística. Pesquisa de orçamentos familiares 2002-2003: Aquisição alimentar domiciliar per capita: Brasil e Grandes Regiões. Rio de Janeiro, 2004a. Disponível em: <http://www.ibge.gov.br/home/estatistica/ populacao/condicaodevida/pof/2002aquisicao/pof2002aquisicao. pdf $>$. Acesso em: 20 ago. 2005.

. Pesquisa de orçamentos familiares 2002-2003: Microdados. Rio de Janeiro, 2004b. 1 CD-ROM.

LOMBARDI-BOCCIA, G. et al. Total-Diet Study: dietary intakes of macro elements and trace elements in Italy. Br. J. Nutr., Cambridge,v. 90, n. 6, p. 1117-1121, 2003. 
Tabela 1. Composição da Cesta de Mercado, segundo compostos de alimentos.

\begin{tabular}{|c|c|c|c|c|c|c|}
\hline Produtos & $\begin{array}{c}\text { Aquisição } \\
\text { per capita/dia (g) }\end{array}$ & $\begin{array}{c}\% \text { no } \\
\text { composto }\end{array}$ & $\begin{array}{l}\text { Quantidade para } 1 \\
\text { kg composto }(\mathrm{g})\end{array}$ & IC & IPC & $\begin{array}{r}\text { Quantidade crua } \\
\text { a ser coletada (g) }\end{array}$ \\
\hline Arroz polido & 65,57 & 88,6 & 886,1 & 2,38 & 1,00 & 372,3 \\
\hline \multirow[t]{2}{*}{ Milho (em grão) } & 3,17 & 4,3 & 42,8 & 1,08 & 2,63 & 104,2 \\
\hline & 74,00 & 100,0 & 1000,0 & & & \\
\hline \multicolumn{7}{|l|}{ Composto 2 - leguminosas } \\
\hline Feijão carioca & 17,78 & 100,0 & 1000,0 & & & \\
\hline \multicolumn{7}{|l|}{ Composto 3 - hortaliças folhosas e florais } \\
\hline Alface & 2,23 & 51,6 & 515,6 & 1,00 & 1,33 & 685,7 \\
\hline \multirow{2}{*}{ Repolho } & 2,09 & 48,4 & 484,4 & 1,00 & 1,72 & 833,2 \\
\hline & 4,32 & 100,0 & 1000,0 & & & \\
\hline \multicolumn{7}{|l|}{ Composto 5 - hortaliças tuberosas e outras } \\
\hline Batata inglesa & 11,09 & 67,2 & 672,5 & 1,03 & 1,06 & 692,1 \\
\hline \multirow{2}{*}{ Cenoura } & 5,40 & 32,8 & 327,5 & 0,90 & 1,17 & 425,8 \\
\hline & 16,49 & 100,0 & 1000,0 & & & \\
\hline \multicolumn{7}{|l|}{ Composto 6 - frutas de clima tropical } \\
\hline Abacaxi & 2,53 & 6,3 & 62,9 & 1,00 & 1,89 & 119,0 \\
\hline Banana nanica & 11,39 & 28,4 & 283,5 & 1,00 & 1,51 & 428,1 \\
\hline Banana prata & 3,96 & 9,9 & 98,6 & 1,00 & 1,51 & 148,9 \\
\hline Laranja pera & 8,31 & 20,7 & 206,8 & 1,00 & 1,57 & 324,6 \\
\hline Mamão & 3,60 & 9,0 & 89,7 & 1,00 & 1,49 & 133,7 \\
\hline Manga & 2,49 & 6,2 & 61,9 & 1,00 & 1,55 & 96,0 \\
\hline Melancia & 7,90 & 19,7 & 196,5 & 1,00 & 2,17 & 426,5 \\
\hline \multicolumn{7}{|l|}{ Composto 9 - massas } \\
\hline Macarrão com ovos & 4,20 & 100,0 & 1000,0 & 2,00 & 1,00 & 500,0 \\
\hline Composto 10 - pães & & & & & & \\
\hline Pão francês & 40,19 & 94,5 & 945,1 & 1,00 & 1,00 & 945,1 \\
\hline Pão de fôrma industrializado de qualquer marca & 2,34 & 5,5 & 54,9 & 1,00 & 1,00 & 54,9 \\
\hline & 42,52 & 100,0 & 1000,0 & & & \\
\hline Composto 11 - biscoitos, roscas, etc. & & & & & & \\
\hline Biscoito doce & 2,64 & 100,0 & 1000,0 & 1,00 & 1,00 & 1000,0 \\
\hline Composto 12 - carnes bovinas de primeira & & & & & & \\
\hline Alcatra & 2,98 & 21,3 & 213,3 & 0,57 & 1,20 & 449,0 \\
\hline Carne de boi de primeira & 2,97 & 21,3 & 213,0 & 0,57 & 1,20 & 448,4 \\
\hline Contra filé & 2,83 & 20,2 & 202,4 & 0,57 & 1,10 & 390,6 \\
\hline Coxão mole (chã de dentro) & 3,09 & 22,2 & 221,5 & 0,57 & 1,10 & 427,5 \\
\hline Patinho & 2,09 & 15,0 & 149,8 & 0,57 & 1,07 & 281,2 \\
\hline & 13,96 & 100,0 & 1000,0 & & & \\
\hline Composto 13 - carnes bovinas de segunda & & & & & & \\
\hline Acém & 3,46 & 20,9 & 208,8 & 0,57 & 1,46 & 534,9 \\
\hline Costela de boi & 4,65 & 28,1 & 280,9 & 0,57 & 1,90 & 936,3 \\
\hline Carne moída de segunda & 3,32 & 20,1 & 200,7 & 0,57 & 1,00 & 352,1 \\
\hline Carne de boi de segunda & 5,12 & 31,0 & 309,5 & 0,57 & 1,46 & 792,8 \\
\hline & 16,54 & 100,0 & 1000,0 & & & \\
\hline Composto 14 - carnes suínas outras & & & & & & \\
\hline Salsicha (varejo) & 2,17 & 100,0 & 1000,0 & 1,00 & 1,00 & 1000,0 \\
\hline
\end{tabular}


Tabela 1. Continuação...

\begin{tabular}{|c|c|c|c|c|c|c|}
\hline Produtos & $\begin{array}{c}\text { Aquisição } \\
\text { per capita/dia (g) }\end{array}$ & $\begin{array}{c}\% \text { no } \\
\text { composto }\end{array}$ & $\begin{array}{l}\text { Quantidade para } 1 \\
\text { kg composto }(\mathrm{g})\end{array}$ & IC & IPC & $\begin{array}{r}\text { Quantidade crua } \\
\text { a ser coletada }(\mathrm{g})\end{array}$ \\
\hline \multicolumn{7}{|l|}{ Composto 15 - carnes de outros animais } \\
\hline Linguiça (varejo) & 4,69 & 100,0 & 1000,0 & 0,80 & 1,00 & 1250,0 \\
\hline Frango congelado & 12,74 & 52,0 & 520,0 & 0,78 & 2,38 & 1586,8 \\
\hline Frango resfriado & 6,18 & 25,2 & 252,4 & 0,78 & 2,38 & 770,2 \\
\hline Peito de galinha ou frango & 2,95 & 12,1 & 120,6 & 0,73 & 1,01 & 166,8 \\
\hline \multicolumn{7}{|l|}{ Composto 17 - leite e creme de leite } \\
\hline Leite de vaca pasteurizado & 75,28 & 54,3 & 543,4 & 1,00 & 1,00 & 543,4 \\
\hline Leite longa vida & 24,56 & 17,7 & 177,3 & 1,00 & 1,00 & 177,3 \\
\hline Leite desnatado & 4,20 & 3,0 & 30,3 & 1,00 & 1,00 & 30,3 \\
\hline Leite esterilizado & 5,28 & 3,8 & 38,1 & 1,00 & 1,00 & 38,1 \\
\hline \multicolumn{7}{|l|}{ Composto 18 - Outros laticínios } \\
\hline Iogurte de qualquer sabor & 6,57 & 100,0 & 1000,0 & 1,00 & 1,00 & 1000,0 \\
\hline \multicolumn{7}{|l|}{ Composto 19 - açúcares } \\
\hline Açúcar refinado & 23,61 & 50,1 & 501,2 & 1,00 & 1,00 & 501,2 \\
\hline Açúcar cristal & 17,64 & 37,5 & 374,5 & 1,00 & 1,00 & 374,5 \\
\hline \multirow[t]{2}{*}{ Açúcar } & 5,85 & 12,4 & 124,2 & 1,00 & 1,00 & 124,2 \\
\hline & 47,10 & 100,0 & 1000,0 & & & \\
\hline \multicolumn{7}{|l|}{ Composto 20 - doces e produtos de confeitaria } \\
\hline Sorvete de qualquer sabor (industrializado) & 2,88 & 100,0 & 1000,0 & 1,00 & 1,00 & 1000,0 \\
\hline \multicolumn{7}{|l|}{ Composto 21 - sais } \\
\hline Sal refinado & 5,93 & 100,0 & 1000,0 & 1,00 & 1,00 & 1000,0 \\
\hline \multicolumn{7}{|l|}{ Composto 25 - bebidas alcóolicas } \\
\hline Cerveja & 17,58 & 100,0 & 1000,0 & 1,00 & 1,00 & 1000,0 \\
\hline \multicolumn{7}{|l|}{ Composto 26 - bebidas não-alcoólicas } \\
\hline Refrigerante de cola & 20,34 & 19,3 & 193,3 & 1,00 & 1,00 & 193,3 \\
\hline Coca-cola & 4,29 & 4,1 & 40,7 & 1,00 & 1,00 & 40,7 \\
\hline Refrigerante de Coca-cola & 4,81 & 4,6 & 45,7 & 1,00 & 1,00 & 45,7 \\
\hline Refrigerante de laranja & 4,23 & 4,0 & 40,1 & 1,00 & 1,00 & 40,1 \\
\hline Refrigerante de guaraná & 32,03 & 30,4 & 304,3 & 1,00 & 1,00 & 304,3 \\
\hline Água mineral & 37,51 & 35,6 & 356,3 & 1,00 & 1,00 & 356,3 \\
\hline Suco de frutas ou vegetais & 2,06 & 2,0 & 19,5 & 1,00 & 1,00 & 19,5 \\
\hline & 105,26 & 100,0 & 1000,0 & & & \\
\hline Composto 27 - cafés & & & & & & \\
\hline Café moído & 7,72 & 100,0 & 1000,0 & 10,00 & 1,00 & 100,0 \\
\hline Composto 28 - alimentos preparados & & & & & & \\
\hline Frango assado ou defumado para viagem & 2,52 & 100,0 & 1000,0 & 1,00 & 1,00 & 1000,0 \\
\hline Composto 29 - peixe de água salgada & & & & & & \\
\hline Peixe inteiro sardinha & 0,38 & 42,8 & 427,5 & 0,64 & 1,50 & 1002,1 \\
\hline Peixe em filé congelado merluza & 0,26 & 28,8 & 287,7 & 0,64 & 1,00 & 449,5 \\
\hline Peixe em filé congelado pescada & 0,25 & 28,5 & 284,8 & 0,64 & 1,00 & 445,0 \\
\hline & 0,89 & 100,0 & 1000,0 & & & \\
\hline Composto 30 - peixe de água doce & & & & & & \\
\hline Peixe inteiro tilápia & 0,24 & 100,0 & 1000,0 & 0,64 & 1,50 & 2343,8 \\
\hline
\end{tabular}


MUNÕZ, O. et al. Estimation of the dietary intake of cadmium, lead mercury, and arsenic by the population of Santiago (Chile) using a Total Diet Study. Food Chem Toxicol., v. 43, p. 1647-1655, 2005. Disponível em: < www.sciencedirect.com/science?_ob >. Acesso em: 30 jan. 2006.

NEW ZEALAND FOOD SAFETY AUTHORITY. New Zealand Total Diet Survey. Introduction. Wellington, 2005. Disponível em: $<$ http://www.nzfsa.govt.nz/science/research-projects/total-dietsurvey/introduction.htm>. Acesso em: 09 jul. 2006.

. New Zealand Total Diet Survey. Report released. Wellington, 2006. Disponível em: http://www.nzfsa.govt.nz/publications/mediareleases/2006-02-24.htm. Acesso em 9 jul. 2006.

. New Zealand Total Diet Survey. 1997/98 Procedures manual. Version 1.0. Wellington, 1997.

ORNELLAS, L. H. Técnica dietética: seleção e preparo de alimentos. 7. ed. rev. e amp. São Paulo: Atheneu, 2001.

PENNINGTON, J. T. Revision of the Total Diet Study food lists and diets. J. Am. Diet. Assoc., Chicago, v. 82, n. 2, p. 166-173, 1983.

PHILIPPI, S. T. Laboratório de técnica dietética: aulas práticas. São Paulo: USP-FSP/HNT, 1999. Manual utilizado como instrumento para aulas práticas da disciplina Técnica Dietética do Curso de Graduação em Nutrição.

. Projetos de pesquisa em Técnica Dietética elaborados pelos alunos do curso de Nutrição. São Paulo: USP-FSP, 1995.

. Técnica dietética: resultados das preparações desenvolvidas em aulas práticas. Faculdade de Saúde Pública, USP. São Paulo: [s.n.], 1994.

PHILIPPI, S. T. et al. Projetos de pesquisa em Técnica Dietética elaborados pelos alunos do curso de Nutrição. São Paulo: USPFSP, 1999.

STENHOUSE, F. The 1992 Australian Market Basket Survey: a Total Diet Survey of pesticides and contaminants. Australian Government Publishing Service. Canberra, 1993.

TURRINI, A.; LOMBARDI-BOCCIA, G. The formulation of the Market Basket of the italian Total Diet 1994-96. Nutr. Res., New York, v. 22, n. 10, p. 1151-1162, Oct. 2002.

U.S. FOOD AND DRUG ADMINISTRATION. Center for Food Safety and Applied Nutrition. Total Diet Study: History and current study design. 2003. Disponível em: <http://www.cfsan.fda.gov/ comm/ tds-hist.html>. Acesso em: 20 out. 2004.

VANNORT, R.; CRESSEY, P.; SILVERS, K. 1997/98 New Zealand Total Diet Survey. Part 2: Elements: selected contaminants \& nutrients. 2000. Disponível em: <http://www.moh.govt.nz/moh.nsf/0/A48 868055568B2814C2568B100823CEF/\$File/ElementsFinal.pdf $>$. Acesso em: 30 jun. 2004.

WHO. World Health Organization. Final draft. Geneva: WHO, 2004. Apresentado ao International Workshop on Total Diet Studies, 3. , 2004, Paris.

. Global Environment Monitoring System. Food contamination monitoring and assessment programme. 1997. Disponível em: <http: //www.who.int/foodsafety/publications/chem/gems/en/ print.html>. Acesso em: 30 jun. 2004.

. Global Environment Monitoring System. Guidelines for the study of dietary intakes of chemical contaminants. Geneva, 1985. (WHO. Offset publication, n. 87).

Programme. Geneva: WHO/Europe and the National Institute of Public Health of the Czech Republic, 2002a. Apresentado ao Sub-Regional Training on Total Diet Studies for Accession Countries, 2002, Brno and Prague, Czech Republic. Disponível em: <http: //www.who.euro.who.int/eprise/main/WHO/Progrs/FOS/ Chemical/20021010_2>. Acesso em: 12 jul. 2004.

Report. Geneva: WHO, 1999. Apresentado ao International Workshop in Cooperation with the Pan American Health Organization: Gems/Food Total Diet Studies- Joint USFDA/ WHO, 1999, Kansas City, K.S. Disponível em: <http//www.who. int/foodsafety/publications/chem./tds_aug1999/en/...>. Acesso em: 30 jun. 2004.

. Report. Geneva: WHO, 2002b. Apresentado ao International Workshop on Total Diet Studies, 2., 2002, Brisbane. Disponível em: $<$ http://www.who.int/foodsafety/publications/chem/tds_feb2002/ em/print.html>. Acesso em: 28 jul. 2004.

. Report. Geneva: WHO, 2002c. Apresentado ao Latin American Total Diet Studies Workshop, 2002, Buenos Aires. Disponível em: $<$ http://www.who.int/foodsafety/publications/chem/tds_july2002>. Acesso em: 30 jun. 2004. 